\title{
Malacofauna límnica da área de influência do lago da usina hidrelétrica de Serra da Mesa, Goiás, Brasil. I. Estudo qualitativo
}

\author{
Silvana C. Thiengo ${ }^{1}$, Sonia B. Santos ${ }^{2} \&$ Monica A. Fernandez ${ }^{1}$ \\ ${ }^{1}$ Departamento de Malacologia, Instituto Oswaldo Cruz. Avenida Brasil 4365, 21045-900 Rio de Janeiro, Rio de Janeiro, \\ Brasil. E-mail: sthiengo@ioc.fiocruz.br; ammon@ioc.fiocruz.br \\ ${ }^{2}$ Laboratório de Malacologia, Instituto de Biologia Roberto Alcantara Gomes, Universidade do Estado do Rio de Janeiro. \\ Rua São Francisco Xavier 524, PHLC, 20550-900 Rio de Janeiro, Rio de Janeiro, Brasil. E-mail: sbsantos@uerj.br
}

\begin{abstract}
Freshwater molluscs of the lake of Serra da Mesa dam, Goiás, Brazil. I. Qualitative study. Schistosomiasis is an important endemic disease associated with environmental change caused by major economic development projects such as the construction of hydroelectric power facilities (dams and the lakes created by them). The difficulty of predicting emerging outbreaks of schistosomiasis associated with these kinds of projects, and the existence of conditions unique to particular projects, necessitate studies in each particular locality in order that appropriate preventive measures can be implemented. The increasing number of dams being built throughout Brazil is a problematic issue because these dams not only create conditions suitable for the establishment of the vector molluscs but also lead to increased permanent human settlement and tourism. The World Health Organization, as well as the Brazilian National Health Foundation, recommend a number of actions to prevent the establishment of schistosomiasis foci, including monitoring mollusc populations. This paper focuses on our work at Serra da Mesa dam, Goiás, where qualitative and quantitative surveys of the freshwater mollusc fauna have been undertaken during the period of 1997 to 2004, aiming mainly to investigate the occurrence and distribution of species of medical importance. Twenty-eight species, belonging to nine families, were found. In addition to documenting new records in the state this paper gives details of the distribution of the two species of medical importance, Biomphalaria straminea (Dunker, 1848) and Lymnaea columella Say, 1817, as well as the invasive freshwater species Corbicula fluminea (Müller, 1774) and Melanoides tuberculatus (Müller, 1774).
\end{abstract}

KEY WORDS. Freshwater molluscs, schistosomiasis vectors, invasive species, distribution.

RESUMO. A esquistossomose é uma importante doença endêmica associada a mudanças ambientais causadas por grandes projetos econômicos, tais como a construção de usinas hidrelétricas. A dificuldade de se prever o surgimento de focos de esquistossomose associados com estes empreendimentos e a existência de condições peculiares em cada tipo de projeto requerem estudos em cada uma dessas áreas, de forma a serem implementadas medidas preventivas adequadas. O número crescente de barragens no país é preocupante pois, além de criarem condições propícias ao desenvolvimento dos moluscos vetores, aumentam a população local e o turismo. Nessas áreas, a Organização Mundial de Saúde e a Fundação Nacional de Saúde recomendam ações preventivas contra a instalação de focos de esquistossomose, dentre elas, o monitoramento das populações de moluscos. Neste trabalho são apresentados dados obtidos por nossa equipe na área da Usina Hidrelétrica de Serra da Mesa (UHSM), Goiás, onde levantamentos qualitativos e quantitativos da malacofauna límnica foram realizados de 1997 a 2004 , com o principal objetivo de investigar a ocorrência e a distribuição das espécies de interesse médico e veterinário. Vinte e oito espécies, pertencentes a nove diferentes famílias foram encontradas. Além de documentar novas ocorrências no Estado, é também apresentada a distribuição de duas espécies de importância médica, Biomphalaria straminea (Dunker, 1848) e Lymnaea columella Say, 1817, bem como de duas espécies exóticas, Corbicula fluminea (Müller, 1774) e Melanoides tuberculatus (Müller, 1774).

PALAVRAS CHAVE. Moluscos límnicos, vetores da esquistossomose, espécies exóticas, distribuição.

A esquistossomose, juntamente com a malária e a febre amarela, são as doenças endêmicas mundialmente importantes associadas a ambientes impactados pelo desenvolvimento de gran- des projetos econômicos, entre eles, a construção de usinas hidrelétricas e barragens em geral (Southgate 1997, Southgate et al. 2001, ZHENG et al. 2002). No Brasil, a crescente demanda 
de energia, associada à imensa disponibilidade de recursos hídricos favoráveis, teve como conseqüência uma grande multiplicação de barragens nos últimos anos, muitas delas na bacia hidrográfica do rio Tocantins. As alterações ambientais trazidas pelas barragens, especialmente mudanças físico-químicas relacionadas à passagem de um sistema lótico para lêntico, afeta profundamente a biodiversidade original (TunDisi et al. 2002) e, no caso específico da esquistossomose, cria condições propícias ao estabelecimento dos moluscos vetores.

Na fase de construção das usinas e barragens, operários oriundos de diversas regiões, muitas delas endêmicas para a esquistossomose, são atraídos pela oportunidade de emprego; na fase de operação, o lago formado estimula atividades de lazer, atraindo permanentemente migrantes e turistas. Essas condições, associadas à contaminação por fezes de indivíduos parasitados, criam os pré-requisitos necessários ao fechamento do ciclo e manutenção da esquistossomose.

A dificuldade de previsão do surgimento de surtos de endemias decorrentes desses empreendimentos e a existência de situações peculiares em cada um deles tornam extremamente necessários estudos preventivos nas áreas afetadas, a tempo de serem executadas as medidas de atenuação possíveis. Nessas áreas submetidas a grandes impactos ambientais e que propiciam, além de intenso fluxo migratório, o assentamento de populações, muitas vezes provenientes de áreas endêmicas de esquistossomose, a Fundação Nacional de Saúde (FNS 1998) recomenda o diagnóstico da situação e a aplicação de medidas preventivas contra a instalação dessa parasitose.

Ao contrário dos estudos existentes sobre a biologia e a composição da fauna de peixes em reservatórios de hidrelétricas no Brasil (AgostinHo et al. 2003, Carvalho \& Silva 1999, TundisI et al. 2002), são escassas as informações sobre os moluscos e especialmente suas interações com os parasitos (SouzA \& Jannotti-Passos 2001, Fernandez \& Thiengo 2002). Assim, de 1997 a 2004, foi efetuado um estudo qualitativo da malacofauna límnica da área de influência do lago da Usina Hidrelétrica de Serra da Mesa (UHSM), localizada no rio Tocantins, em Minaçu

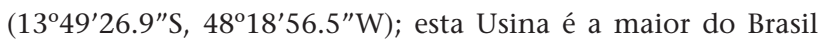
em volume de água represada, com $1.784 \mathrm{~km}^{2}$ de área de superfície, atingindo oito municípios (Barro Alto, Campinaçu, Campinorte, Colinas do Sul, Minaçu, Niquelândia, Santa Rita do Novo Destino e Uruaçu). Os principais objetivos do presente estudo foram identificar a malacofauna presente na área, de modo a fornecer subsídios para estudos epidemiológicos e parasitológicos, e contribuir para um melhor entendimento das alterações das comunidades malacológicas frente às mudanças ambientais. Neste trabalho são apresentados os resultados do levantamento dos moluscos límnicos realizado neste período, nos oito municípios acima citados, a distribuição das duas espécies de importância médico-veterinária, Biomphalaria straminea (Dunker, 1848) e Lymnaea columella Say, 1817, bem como das espécies invasoras Corbicula fluminea (Müller, 1774) e Melanoides tuberculatus (Müller, 1774).

\section{MATERIAL E MÉTODOS}

A área de estudo compreendeu os oito municípios sob influência do lago da Usina Hidrelétrica de Serra da Mesa (UHSM) (Fig. 1), contemplando principalmente as áreas entorno do reservatório, embora tenham sido realizadas coletas em todos os distritos de cada município, bem como nas principais localidades (Tabs I e II).

Em abril de 1996, antes do enchimento do reservatório, foi feita uma visita ao local para o reconhecimento da área, possibilitando a realização das primeiras coletas. Posteriormente, coletas sistemáticas foram efetuadas nos seguintes meses: abril e outubro de 1997; março, abril, junho, setembro e novembro de 1998; janeiro, março, maio e novembro de 1999; março, maio, junho, setembro e novembro de 2000; janeiro, março, maio, junho, setembro e novembro de 2001; janeiro, março, maio, julho, agosto e novembro de 2002; março, maio, setembro e novembro de 2003; maio e setembro de 2004. Entre 2000 e 2003 foi realizada, simultaneamente ao estudo qualitativo, uma análise quantitativa das espécies de importância médico-veterinária, e em 2004 foram feitas coletas complementares na área.

As coletas foram realizadas em biótopos favoráveis à ocorrência de moluscos límnicos, tais como rios, riachos, lagos, brejos, valas de esgoto e de drenagem, caixas d'água e bebedouros de animais, tanto a montante quanto a jusante da barragem. Os moluscos foram coletados com auxílio de conchas de captura, colocados em potes plásticos contendo água do criadouro e etiquetados. Um lote representativo de cada amostra foi sacrificado, após relaxamento em solução de Hypnol a 0,05\% durante $6 \mathrm{~h}$ e, fixados em solução de Railliet-Henry. O restante foi acondicionado para o transporte até o Departamento de Malacologia do IOC/FIOCRUZ, onde foram mantidos em aquários e, em intervalos de cinco dias, durante um mês, foram expostos à luz para pesquisa de larvas de trematódeos, principalmente de Schistosoma mansoni Sambon, 1907 e de Fasciola hepatica (Linneu, 1758). Os detalhes técnicos são fornecidos em Thiengo (1995a) e Boaventura et al. (2002).

A identificação foi feita através da conquiliologia e de dissecções anatômicas dos exemplares fixados de cada amostra.

Todo o material de interesse taxonômico e zoogeográfico está depositado na Coleção Malacológica do Instituto Oswaldo Cruz, Fundação Instituto Oswaldo Cruz, Rio de Janeiro, Brasil.

\section{RESULTADOS}

Foram obtidas 1.373 amostras durante todo o período sendo nove em 1996, 71 em 1997, 142 em 1998, 155 em 1999, 179 em 2000, 212 em 2001, 258 em 2002, 242 em 2003 e 105 em 2004. Dessas amostras, nove foram obtidas antes do barramento do rio Tocantins (ocorrido em outubro de 1996), 104 durante o período de enchimento do reservatório até o início da operação da UHSM (abril de 1998) e 1.260 no período de pós-enchimento, o que possibilitou acompanhar as altera- 


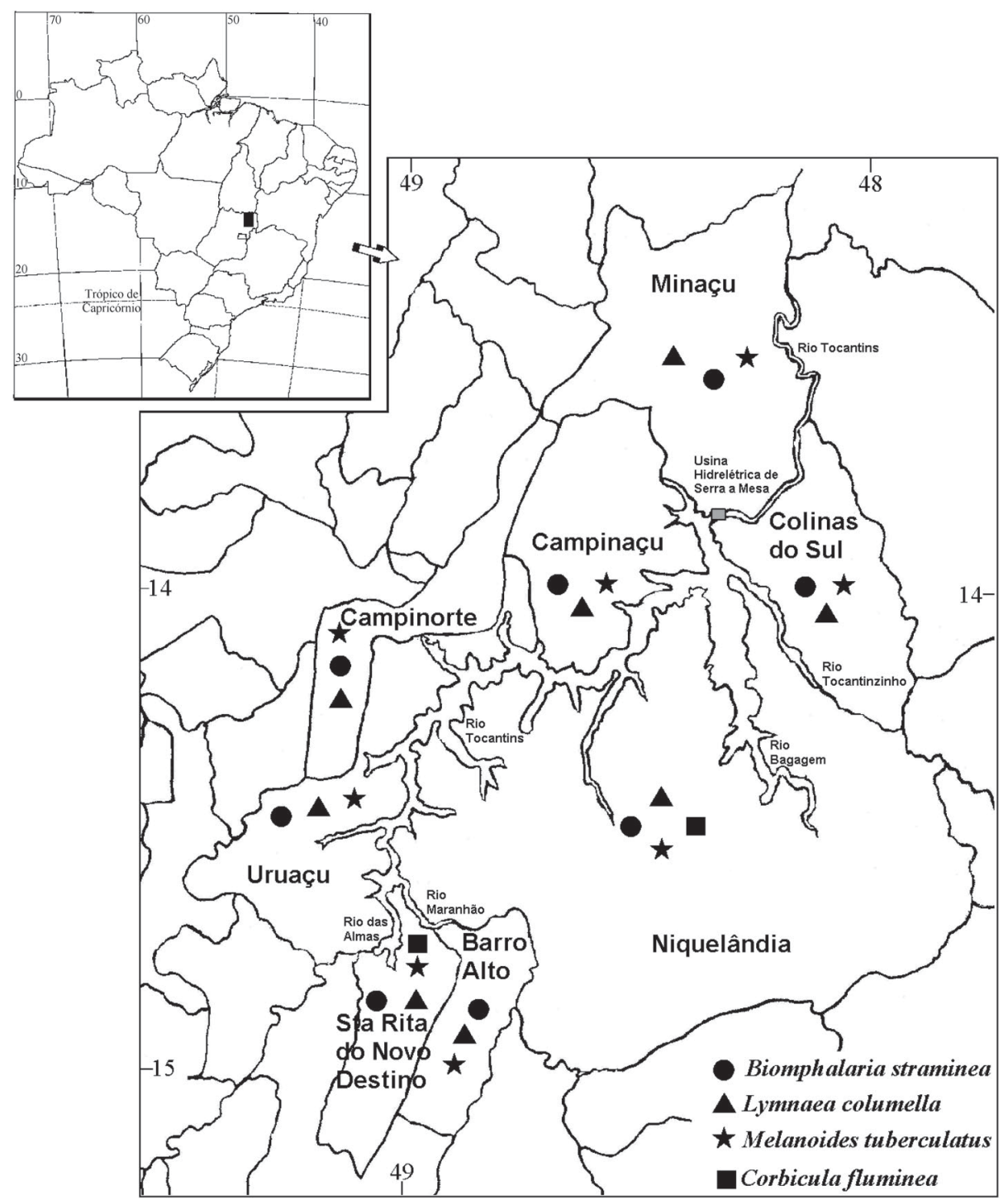

Figura 1. Área de estudo com a localização das espécies de importância médico-veterinária e das espécies exóticas.

ções ocorridas na malacofauna a medida em que as modificações ambientais trazidas pelas diferentes etapas da construção da UHSM foram acontecendo.

As espécies, pertencentes às famílias Ampullariidae, Ancylidae, Corbiculidae, Hydrobiidae, Physidae, Planorbidae, Lymnaeidae, Sphaeriidae e Thiaridae, bem como as localidades onde foram registradas encontram-se nas tabelas I e II. A distribuição das espécies de importância médico-veterinária, $B$. straminea e L. columella, bem como dos moluscos exóticos $C$. fluminea e M. tuberculatus, são apresentadas na figura 1.

Foram encontradas as seguintes espécies: Antillorbis nordestensis (Lucena, 1954); Aylacostoma tenuilabris (Reeve, 1860); Biomphalaria intermedia (Paraense \& Deslandes, 1962); B. straminea; Biomphalaria occidentalis Paraense, 1981; Biomphalaria schrammi (Crosse, 1864); Burnupia ingae Lanzer, 1991; C. fluminea; Drepanotrema anatinum (Orbigny, 1835); Drepanotrema cimex (Moricand, 1839); Drepanotrema depressissimum (Moricand, 1839); Drepanotrema lucidum (Pfeiffer, 1839); Eupera simoni (Jousseaume, 1889); Eupera tumida (Clessin, 1879); Ferrissia gentilis Lanzer; 1991; Gundlachia radiata (Guilding, 1828); Gundlachia ticaga (Marcus \& Marcus, 1962); Idiopyrgus souleyetianus Pilsbry, 1911; Laevapex fuscus Adams, 1841; L. columella; M. tuberculatus; Physa marmorata Guilding, 1828; Pisidium bejumae Baker, 1930; Pisidium punctiferum (Guppy, 1867); Pomacea canaliculata (Lamarck, 1822); Pomacea figulina (Spix, 1827); Pomacea lineata (Spix, 1827) e Uncancylus concentricus (Orbigny, 1835). 


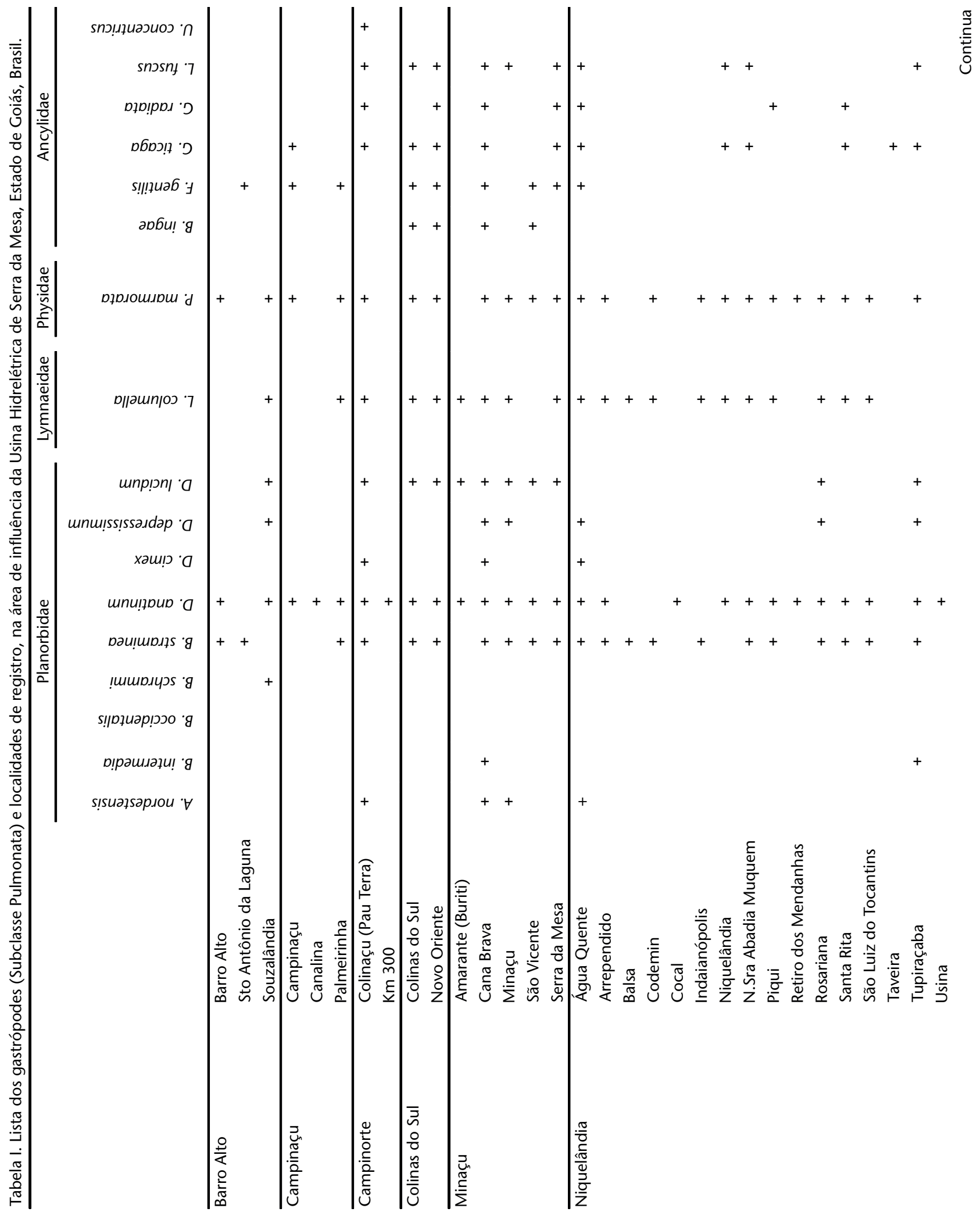

Revista Brasileira de Zoologia 22 (4): 867-874, dezembro 2005 


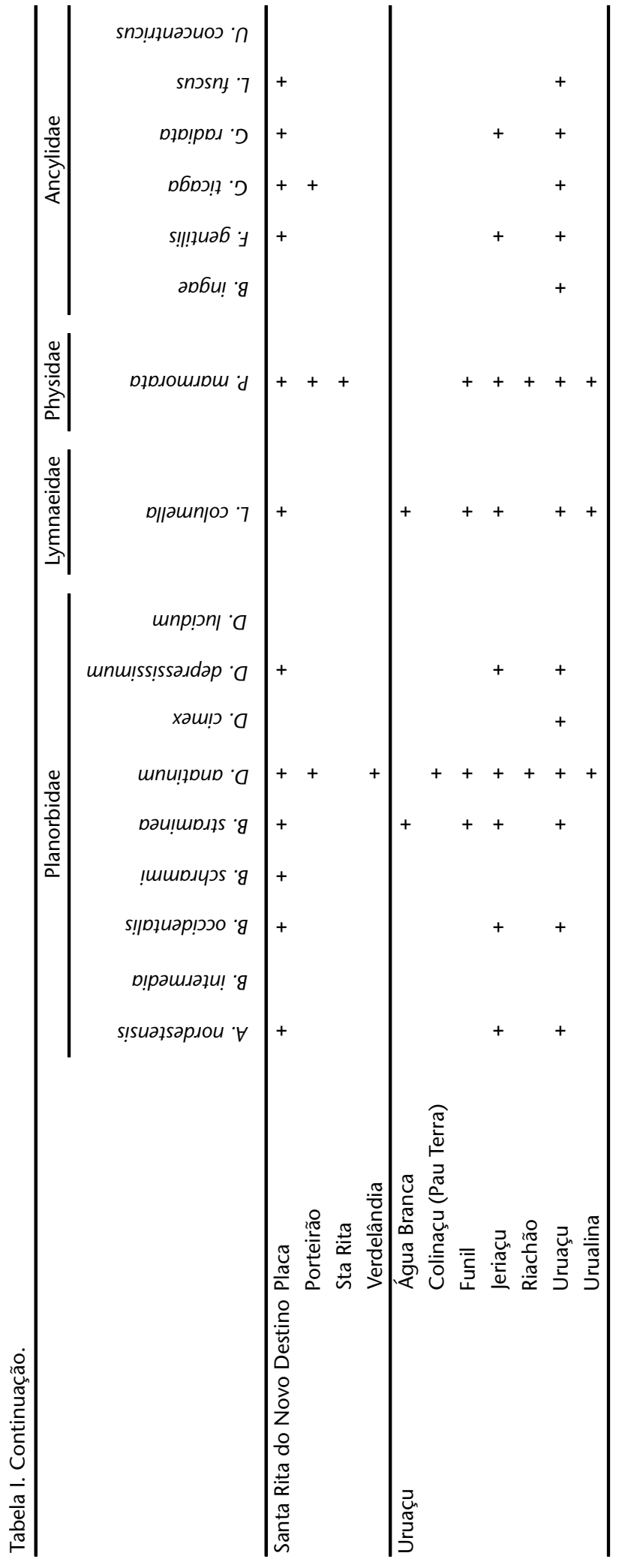

Em abril de 1996, B. straminea, espécie vetora da esquistossomose, foi encontrada em remansos do rio Bagagem, no município de Niquelândia. As primeiras espécies a colonizarem o lago, G. ticaga, D. anatinum e L. columella, foram observadas em abril de 1998, em pequenos troncos e galhos carreados dos rios e riachos represados pela barragem em diferentes pontos de coleta. Quanto à B. straminea, embora tenha sido registrada na mesma campanha habitando a área do reservatório, sua distribuição restringia-se a poucas fazendas presentes na margem do lago. Simpatria entre $B$. straminea e $B$. occidentalis foi observada somente no município de Uruaçu, às margens do reservatório, em novembro de 1998. Entre 2000 e 2002, a ocorrência de $B$. straminea e $M$. tuberculatus às margens do reservatório foi constante, em simpatria com L. columella ou P. marmorata. Já em 2003 e 2004, foi notável a diminuição das populações de $M$. tuberculatus, o que não ocorreu em relação à $B$. straminea. Algumas espécies, como $A$. tenuilabris e $D$. anatinum, registradas freqüentemente no rio Tocantins antes da formação do lago, tornaram-se mais escassas. Por outro lado, a partir de 2002, houve uma intensa colonização por G. radiata, substituindo G. ticaga.

Os dados ecológicos envolvendo a riqueza e a sucessão das espécies, a freqüência de ocorrência e a constância de determinada espécie, e a similaridade da fauna nos inúmeros ambientes criados após a transformação do ambiente lótico em lêntico, encontram-se em fase de análise.

Embora não tenham sido detectadas formas larvais de $S$. mansoni ou $F$. hepatica, estádios larvais de outras espécies de trematódeos foram observados em B. straminea, D. cimex, D. depressissimum, D. lucidum, G. radiata, M. tuberculatus, P. figulina e $P$. lineata. As diferentes interações molusco-parasito observadas, muitas das quais inéditas, encontram-se em fase de publicação.

\section{DISCUSSÃO}

Estudos envolvendo a fauna malacológica em áreas impactadas por grandes projetos econômicos são raros no Brasil. Os resultados do presente trabalho permitiram registrar quando e como surgiram as primeiras populações de $B$. straminea no reservatório criado com a construção da UHSM e, após sua dispersão, o estabelecimento das populações nos oito municípios presentes na área de influência desta Usina Hidrelétrica. Dentre as três espécies vetoras do $S$. mansoni no Brasil, esta espécie é a que possui a mais ampla distribuição geográfica e maior adaptação a todas as variedades de clima e condições ecológicas, ocorrendo de norte a sul do país, com exceção de Rondônia e Amapá (PARAEnse 1986, 2001).

Sob o ponto de vista epidemiológico, deve-se ressaltar que $B$. straminea é considerada má hospedeira de $S$. mansoni, apesar de ser um excelente transmissor capaz de manter altos índices de infecção na população humana, visto que é comum o encontro de uma taxa de infecção natural menor do que 0,01\% nos moluscos, após o exame de amostras superiores a 5.000 exemplares provenientes de locais onde as taxas de in- 
Tabela II. Lista dos bivalves e gastrópodes (Subclasse Prosobranchia) e localidades de registro, na área de influência da Usina Hidrelétrica de Serra da Mesa, Estado de Goiás, Brasil.

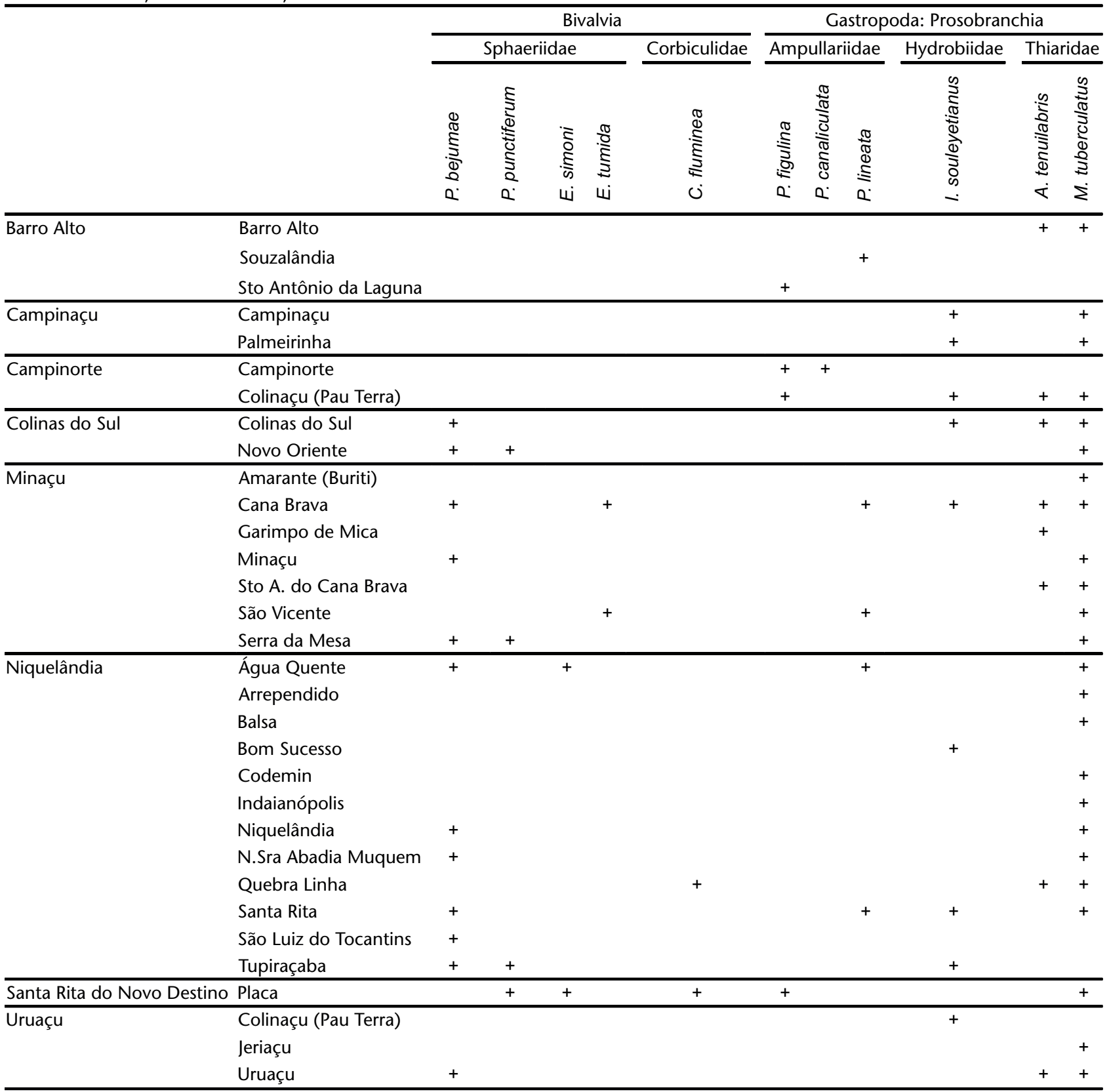

fecção humana estão em torno de 60\% (PARAENSE \& CoRrêA 1989). Deve-se também considerar que a expansão da esquistossomose é um processo lento e contínuo, sendo os primeiros casos em novas localidades quase sempre descobertos casualmente em exames de fezes, estando nesse momento a infecção já radicada nas populações dos hospedeiros intermediários e definitivos, depois de ter realizado, através de longo período, uma sucessão de ciclos entre o molusco e o homem, até atingir uma densida- de detectável a exames aleatórios (PARAEnse 1986). Diante destes fatores, estudos de suscetibilidade das colônias de $B$. straminea provenientes da área de influência da UHSM foram realizados (FernANdez \& THIENGo 2002), os quais demonstraram a possibilidade desta espécie vir a atuar como vetora da esquistossomose na referida área.

Além de $B$. straminea, é importante ressaltar a ocorrência de B. occidentalis na área investigada pois, embora refratária ao 
S. mansoni, é facilmente confundida com outro vetor que não ocorre na região, Biomphalaria tenagophila (Orbigny, 1835), devido a grande semelhança existente entre as conchas das duas espécies.

Nos últimos anos, a crescente introdução e estabelecimento de moluscos exóticos no país, tem sido um motivo de grande preocupação entre os ambientalistas. Sob este aspecto, a presença do bivalve asiático C. fluminea e do gastrópode $M$. tuberculatus na área em estudo merece destaque. O primeiro, encontrado no rio das Almas, foi registrado pela primeira vez no Brasil, na bacia do rio Guaíba e do Jacuí, Rio Grande do Sul, provavelmente introduzido no início da década de 1970 (VeITENHEIMER-Mendes 1981). Esta espécie, por formar colônias em grandes densidades, pode provocar o declínio das populações de bivalves nativos, como Sphaeriidae, além de causar o entupimento de tubulações e trocadores de calor, causando prejuízos econômicos às usinas hidrelétricas, como o ocorrido na UHE de Porto Colômbia, no rio Grande, em Minas Gerais (Mansur et al. 2004).

Com relação a M. tuberculatus, em apenas cinco anos após o barramento do rio Tocantins, densas populações puderam ser observadas em extensas áreas nas margens do reservatório da UHSM, o que comprova sua característica de espécie invasora. Paralelamente observou-se uma grande redução do número de exemplares da espécie nativa $A$. tenuilabris, nos rios Almas, Bagagem e Cana Brava, perante o aumento exponencial de populações de $M$. tuberculatus. A distribuição dessa espécie no país, atualmente presente em 16 estados, bem como suas implicações com a malacofauna nativa foram fornecidas por FERNANDEZ et al. (2003).

Como a pecuária constitui uma das principais atividades econômicas em Goiás, a extensa distribuição de populações de L. columella nas margens do reservatório da UH Serra da Mesa, associada ao estreito contato com os rebanhos bovinos mantidos próximos ao reservatório, é preocupante pela possibilidade de introdução da fasciolose na região.

Em relação aos demais planorbídeos este estudo confirmou a ampla distribuição geográfica de $A$. nordestensis, $B$. schrammi, D. anatinum, D. cimex, D. depressissimum e D. lucidum, assinalada por Paraense (1975). Quanto a B. intermedia, espécie de distribuição mais restrita, os dados permitiram ampliar a sua distribuição, anteriormente com ocorrências em São Paulo, Mato Grosso do Sul e Minas Gerais (Paraense 1975, 1985, Souza et al. 2001).

Quanto aos ampulariídeos, este constitui o primeiro relato de $P$. canaliculata, $P$. figulina e $P$. lineata para Goiás. Dentre essas espécies, $P$. canaliculata é a que possui a maior distribuição geográfica e por apresentar um grande polimorfismo, provavelmente, constitui um complexo de espécies (THiengo 1995b). Outras novas ocorrências foram encontradas em Ancylidae, onde as espécies $B$. ingae e $G$. ticaga tiveram sua distribuição ampliada para a região Centro-Oeste. Restritas anteriormente às regiões Sul e Sudeste, respectivamente, $B$. ingae foi descrita para os Estados de Santa Catarina e Rio Grande do
Sul (LANZER 1991), enquanto G. ticaga possui registros desde o Sudeste do Brasil até a Argentina (Lanzer 1996, SANTos 2003). A presença de $L$. fuscus, com várias citações genéricas no Brasil (LANZer 1996, SANTos 2003) amplia a distribuição do gênero Laevapex Walker, 1903 para a região Centro-Oeste.

A presença de populações bem estabelecidas de $B$. straminea no lago da UHSM e o estreito contato da população com o mesmo, aliado ao quase desconhecimento do impacto provocado pela construção de hidrelétricas sobre a dinâmica populacional desses vetores, demonstram a necessidade da adoção de abordagens multidisciplinares em saúde e educação nessas áreas, visando à prevenção da instalação de focos de esquistossomose e de outras parasitoses veiculadas pela água.

\section{AGRADECIMENTOS}

A Furnas Centrais Elétricas S.A., em especial ao Engenheiro Pedro Augusto C. Araújo, e a Serra da Mesa Energia S.A., pelo apoio logístico na realização dos trabalhos de campo. A Dra Maria Cristina Dreher Mansur e a MSc Maria Cristina Pons da Silva pela identificação dos bivalves e dos hidrobiídeos, respectivamente; e às Biólogas Maria Fernanda Boaventura e Aline Carvalho de Mattos pela assistência técnica. A Moisés de Souza Oliveira, Ariovaldo Gomes Coelho e Chisto Soares da Silva pela valiosa colaboração durante as atividades de campo.

\section{REFERÊNCIAS BIBLIOGRÁFICAS}

Agostinho, A.A.; L.C. Gomes \& H.F. Julio Jr. 2003. Relações entre macrófitas aquáticas e fauna de peixes, p. 261-279. In: S.M. TномаZ \& L.M. Bini (Eds). Ecologia e manejo de macrófitas aquáticas. Maringá, Eduem, 341p.

Boaventura, M.F.; M.A. Fernandez; S.C. Thiengo; R.E. Silva \& A.L. Melo. 2002. Formas larvais de Trematoda provenientes de gastrópodes límnicos da microrregião Rio de Janeiro, sudeste do Brasil. Lundiana, Belo Horizonte, 3 (1): 45-49.

Carvalho, E.D. \& V.F.B. Silva. 1999. Aspectos ecológicos da ictiofauna e da produção pesqueira no Reservatório de Jurumirim, p. 771-799. In: R. HenRY (Ed.). Ecologia de reservatórios: estrutura, função e aspectos Sociais. Botucatu, Fundibio, Fapesp, 800p.

Fernandez, M.A. \& S.C. Thiengo. 2002. Susceptibility of Biomphalaria straminea (Dunker, 1848) from Serra da Mesa Dam, Goiás, Brazil to infection with three strains of Schistosoma mansoni Sambon, 1907. Memórias do Instituto Oswaldo Cruz, Rio de Janeiro, 97 (Suppl. I): 59-60.

Fernandez, M.A.; S.C. Thiengo \& L.R.L. Simone. 2003. Distribution of the introduced freshwater snail Melanoides tuberculatus (Gastropoda: Thiaridae) in Brazil. The Nautilus, Flórida, 117 (3): 78-82.

FNs. 1998. Controle da esquistossomose: diretrizes técnicas. Brasília, Fundação Nacional de Saúde, $2^{a}$ ed., 70p.

Lanzer, R.M. 1991. Duas novas espécies de Ancylidae (Gastropoda: Basommatophora) para o sul do Brasil. Revis- 
ta Brasileira de Biologia, Rio de Janeiro, 51 (4): 703-719.

LANZER, R.M. 1996. Ancylidae (Gastropoda, Basommatophora) na América do sul: sistemática e distribuição. Revista Brasileira de Zoologia, Curitiba, 13 (1): 175-210.

Mansur, M.C.D.; C.T. Callil; F.R. Cardoso \& J.A.A. Ibarra. 2004. Uma retrospectiva e mapeamento da invasão de espécies de Corbicula (Mollusca, Bivalvia, Veneroida, Corbiculidae) oriundas do sudeste Asiático, na América do Sul, p. 39-58. In: J.S.V. Silva \& R.C.C.L. Souza (Eds). Água de Lastro e Bioinvasão. Rio de Janeiro, Editora Interciência, 224p.

Paraense, W.L. 1975. Estado atual da sistemática dos planorbídeos brasileiros. Arquivos do Museu Nacional, Rio de Janeiro, 55: 105-128.

Paraense, W.L. 1985. Biomphalaria intermedia in Mato Grosso do Sul, Brazil, and Misiones, Argentina (Pulmonata: Planorbidae). Memórias do Instituto Oswaldo Cruz, Rio de Janeiro, 80 (2): 247-250.

Paraense, W.L. 1986. Distribuição dos caramujos no Brasil. Anais da Academia Mineira de Medicina, Belo Horizonte, (Suplemento de 1983-1984): 117-128.

Paraense, W.L. 2001. The schistosome vectors in the Americas. Memórias do Instituto Oswaldo Cruz, Rio de Janeiro, 96 (Suppl.): 7-16.

Paraense W.L. \& Lr CorrêA. 1989. A potential vector of Schistosoma mansoni in Uruguay. Memórias do Instituto Oswaldo Cruz, Rio de Janeiro, 84 (3): 281-288.

SANTos, S.B. 2003. Estado atual do conhecimento dos ancilídeos na América do Sul (Mollusca: Gastropoda: Pulmonata: Basommatophora). Revista de Biologia Tropical, Costa Rica, 51 (Suppl. 3): 191-224.

SOUTHGATE, V.R. 1997. Schistosomiasis in the Senegal river basin: before and after the construction of the dams at Diama, Senegal and Manantali, Mali and future prospects. Journal of Helminthology, Wallingford, 71 (2): 125-132.

Southgate VR; L.A.T. Tchuente; M. Sene; D. De ClercQ; A. Theron;
J. Jourdane; B.L. Webster; D. Rollinson; B. Gryseels \& J. VercruYsSe. 2001. Studies on the biology of schistosomiasis with emphasis on the Senegal River basin. Memórias do Instituto Oswaldo Cruz, Rio de Janeiro, 96 (Suppl.): 75-78.

SouZA, C.P. \& L. JanNotTI-PAssos. 2001. Resistance of Biomphalaria occidentalis from Varzae das Flores dam, Minas Gerais, to Schistosoma mansoni infection detected by low stringency polymerase chain reaction. Memórias do Instituto Oswaldo Cruz, Rio de Janeiro, 96 (3): 381-383.

Souza, C.P.; R.L. Caldeira; S.C. Drummond; A.L. Melo; C.T. GuiMarÃes \& O.S. CaRvalho. 2001. Geographical distribution of Biomphalaria snails in the State of Minas Gerais, Brazil. Memórias do Instituto Oswaldo Cruz, Rio de Janeiro, 96 (3): 293-302.

Thiengo, S.C. 1995a. Técnicas malacológicas, p. 255-265. In: F.S. Barbosa (Ed.). Tópicos em Malacologia Médica. Rio de Janeiro, Editora Fiocruz, 314p.

Thiengo, S.C. 1995b. Família Pilidae Connoly, 1927 (Ampullariidae Gray, 1824), p. 50-69. In: F.S. Barbosa (Ed.). Tópicos em Malacologia Médica. Rio de Janeiro, Editora Fiocruz, $314 \mathrm{p}$.

TundisI, J.G.; T.M. Tundisi \& O. Rocha. 2002. Ecossistemas de águas interiores, p. 153-170. In: A.C. Rebouças \& B. BRAGA (Eds). Águas doces no Brasil. Capital ecológico, uso e conservação. São Paulo, Escrituras, $2^{\mathrm{a}}$ ed., 704p.

Veitenheimer-Mendes, I.L. 1981. Corbicula manilensis (Philippi, 1844) molusco asiático, na bacia do Jacuí e do Guaíba, Rio Grande do Sul, Brasil (Bivalvia, Corbiculidae). Iheringia, Série Zoologia, Porto Alegre, 60: 63-74.

Zheng, J; X.G. Gu; Y.L. Yu; J.H. Ge; X.X. YANG; C.H. He; C. TANG; K.P. CAI; Q.W. JiANG; Y.S. Liang; T.P. WANG; X.J. Xu; J.H. ZHONG; H.C. YUAN \& X.N. Zhou. 2002. Relationships between the transmission of Schistosomiasis japonica and the construction of the Three Gorge Reservoir. Acta Tropica, Stockholm, 82 (2): 147-156

Recebido em 01.IV.2005; aceito em 30.IX.2005. 